\title{
Inducción, Deducción y Fenomenología en la Investigación Psicológica Sobre Casos de Violencia Familiar
}

\author{
Induction, Deduction and Phenomenology in Psychological Research on \\ Cases of Family Violence
}

\author{
Hugo Manuel Sánchez Jiménez \\ Centro Emergencia Mujer Huacho
}

Recibido: $19-05-21$

Aceptado: $26-10-21$

Publicado: $23-12-21$

\section{Resumen}

En el presente artículo se enfatiza la importancia de los métodos inductivo, deductivo y fenomenológico que intervienen en la práctica e investigación de la Psicología forense, teniendo en cuenta el estado actual de las investigaciones en nuestro país en la temática de la violencia familiar. El avance del fenómeno de la violencia en nuestra sociedad exige profundizar en la investigación y realizar un correcto diagnóstico de los efectos de la violencia; es por ello que conocer sobre el empleo y las bases epistemológicas que demandan estos tres métodos, permitirá a los investigadores de la profesión afianzarse en la intervención integral de dichos casos.

Palabras clave: Inducción; deducción; fenomenología; violencia familiar; victimología.

\begin{abstract}
This article emphasizes the importance of inductive, deductive and phenomenological methods that intervene in the practice and investigation of forensic psychology, taking into account the current state of investigations in our country on the subject of family violence. The advance of the phenomenon of violence in our society requires deepening research and making a correct diagnosis of the effects of violence; that is why knowing about employment and the epistemological bases required by these three methods, will allow researchers in the profession, to gain a foothold in the comprehensive intervention of such cases.
\end{abstract}

Keywords: Induction; deduction; phenomenology; family violence; victimology.

\footnotetext{
1 Centro Emergencia Mujer Huacho -Programa Nacional Contra la Violencia Familiar y Sexual, Huacho, Perú. Autor para correspondencia: hugomsj@hotmail.com ORCID: https://orcid.org/0000-0002-1997-3844

(C) Los autores. Este artículo es publicado por la Revista de Investigación en Psicología de la Facultad de Psicología, Universidad Nacional Mayor de San Marcos. Este es un artículo de acceso abierto, distribuido bajo los términos de la licencia Creative Commons Atribución 4.0 Internacional (CC BY 4.0) [https://creativecommons.org/licenses/by/4.0/deed.es] que permite el uso, distribución y reproducción en cualquier medio, siempre que la obra original sea debidamente citada de su fuente original.
} 


\section{INTRODUCCIÓN}

La psicología, para mantener su status de ciencia a través de la investigación, y así conformar nuevos conocimientos, leyes y teorías, debe importar cierta postura epistemológica de la filosofía clásica, la cual sostiene que cuando se trata de determinar si un conocimiento puede formar parte de la ciencia o no, se debe distinguir entre su contexto de descubrimiento y el de justificación o validación del cual surge (Reichenbach, 1938, citado por Gonzales, 2006). De este modo, al abocarnos al contexto de justificación del conocimiento científico, encontramos que Popper considera que la labor del científico es la de formular teorías y ponerlas a prueba, mas no así el acto de concebir o inventar una teoría, que puede ser más bien de interés para la psicología empírica, y que, por ende, no podría ser materia de análisis lógico (Popper, 1980).

Hanson (1977, citado por Gonzales, 2006) explica que no se trata de desarrollar una teoría a partir de los hallazgos basados en casos particulares, sino de inventarla; considera que una teoría juiciosa es aquella que explica hechos problemáticos, donde también participa la inducción que genera teoría sobre la base de problemas previos. Dicho de otro modo, se formula una hipótesis porque detrás hay un problema que resolver y plantearla no es un proceso lineal; este método parte de nociones específicas a otras más generales, en dependencia a tiempos, territorios y temas, tal como ocurre con los preceptos de la "teoría fundamentada" (Gonzales, 2006).

Así mismo, en nuestro medio, en cuanto a la caracterización de los trabajos de investigación sobre violencia familiar, investigaciones previas destacan el empleo, en mayor medida, del enfoque cuantitativo (66.6\%) sobre el cualitativo (17.46\%); siendo los diseños empleados: descriptivo, correlacional y la mixtura de ambos (34.86\%), diseño descriptivo-comparativo (28.57\%), estudio de casos (6.30\%), cuasi experimental (3.17\%), así como descriptivo-explicativo (1.60\%), entre los más utilizados (Chávez et al., 2017). Por lo que dicha producción científica psicológica se deriva del empleo de los siguientes métodos: inductivo, deductivo y fenomenológico. El método inductivo consiste en la operación lógica de obtener una conclusión partiendo de los hechos a las leyes, de los casos particulares a postulados más generales; mientras que el método deductivo tiene reglas mediante las cuales se parte de uno o varios conocimientos ya adquiridos (o hipótesis) para arribar a una conclusión particular (Dorna, 1985). Dávila (2006) explica sobre el razonamiento inductivo que: "según Bacon, para obtener conocimiento es imprescindible observar la naturaleza, reunir datos particulares y hacer generalizaciones a partir de ellos" (p. 185). De otro lado, sobre el razonamiento deductivo el autor sostiene que: "las conclusiones deductivas son necesariamente inferencias hechas a partir de un conocimiento que ya existía” (p. 185). 
Con relación a la fenomenología, Escalante y Páramo (2011, citados por Gil et al., 2011, p. 160) refieren que: "la fenomenología quiere decir hacer ver lo que se muestra (fenómeno) tal como se muestra. Lo que se intenta es comprender la narración del sujeto, desde la búsqueda de lo dado en la experiencia sensible". Por otro lado, la diferencia entre psicología y fenomenología también las asemeja: una y otra trabajan sobre lo netamente subjetivo; una, en sus manifestaciones naturales y espontáneas; y la otra, en su actitud pensante y consciente (Vargas, 2011).

De otro lado, en cuanto al fenómeno de la violencia y, por ende, el de la investigación en victimología, se sabe que su estudio está inmerso en el campo de la psicología forense, la cual tiene algunos hitos desde su aparición: Münsterberg, discípulo de Wundt, consideró en 1862 que la psicología ha de desarrollarse en campos como educación, industria, salud y justicia; en 1908 su libro On the witness Stand se convirtió en un hito de la psicología jurídica. A su vez, en el siglo XIX empiezan a tomar realce la psicología jurídica (colectiva-teórica) y la psicología forense (individual y aplicada). Y es en el siglo XX, específicamente entre los años 50 y 60 , cuando el psicólogo asume su rol de perito o experto en las audiencias. Mientras que en los años 80 y 90 se instauran los centros de formación, y a su vez el papel del psicólogo adopta el nivel de auxiliar judicial que aporta científicamente al derecho penal. Y en tiempos actuales la psicología forense, como área de la psicología, es la que ha logrado un mayor desarrollo (Grijalva, 2015).

\section{De la inducción a la deducción}

Como hecho histórico y ejemplificante apreciamos que, durante el desarrollo precientífico de la medicina, Hildegarda de Bingen (Alemania, 1098-1179) tuvo una vasta producción como médico, naturalista y también como teóloga; su obra Physica está basada en la observación de la naturaleza animal, vegetal y mineral, y en Causae et Curae se ciñe a los orígenes y tratamiento de las enfermedades. Sus conocimientos los adquirió en su práctica curativa, y algunos de ellos posteriormente fueron verificados por la ciencia moderna. Una vez ya con la práctica, sus teorías y tratamientos alcanzan experticia y empieza a distanciarse de las concepciones médicas tradicionales, concibiendo que los elementos de la naturaleza sirven por sus propiedades innatas, pues son beneficiosos para la salud (Barceló, 2016). En base a su experiencia acopia información valiosa para los tratamientos medicinales de la época, empleando la evidencia empírica de sus curaciones como sustento del desarrollo inductivo de la ciencia.

También tenemos que el desarrollo de la lógica inductiva de Aristóteles se inició a finales del siglo XII (Gonzales, 2006). Al respecto, Lakatos (1978, 1981, citado por Gonzales, 2006), considera que la confianza en la inducción como garante del conocimiento se empezó a perder desde el siglo XIII y que se perdió del todo desde el siglo XVIII debido al desarrollo de las geometrías no euclidianas. 
$\mathrm{Y}$ es en el siglo XX, bajo la influencia de Popper y su falsacionismo, que en el Círculo de Viena se relega el enfoque inductivo para dar paso al hipotético-deductivo. No obstante, el enfoque cualitativo, originado en la Escuela de Frankfurt, rescató el inductivismo para el estudio de los hechos sociales (Echevarría, 1999; Villena, 2014; citados por Sánchez, 2019). Surge, pues, como una reacción de protesta frente a las operaciones deductivas que no explican todos los fenómenos sociales (Sánchez, 2019).

Popper (1980) considera que si queremos justificar las inferencias inductivas se debe plantear un principio de inducción. Pero dicho principio se basa en el criterio de que la naturaleza es regular -principio que Popper censura-, pues al considerarse que todo acontecimiento determinado ocurre bajo criterios de una regularidad universal, implicaría admitir su carácter de no falsabilidad, requisito básico para él para que un conocimiento sea admitido como constitutivo de la ciencia (Popper, 1980). Por otro lado, la inducción es un proceso que implica un "salto inductivo", es decir, el pasar de observaciones a generalizaciones (Gonzales, 2006). Tal como ocurre con los inductivistas, que, cuando encuentran que el cisne 1 es blanco, el cisne 2 y 3 también lo son, y así sucesivamente de forma infinita; llegan, de este modo, a la conclusión: "todos los cisnes son blancos", o sea, pasan a concluir de casos observados a los no observados, generalizando hacia la totalidad de los miembros de dicha especie (Popper, 1980). Popper, pues, es contrario a la ideología de los empiristas lógicos, niega dicho principio y sostiene que "es superfluo todo principio de inducción, y que lleva forzosamente a incoherencias (incompatibilidades) lógicas" (Popper, 1980 p. 28).

De este modo, Popper crítica al inductivismo la imposibilidad de justificar lógicamente sus razonamientos, los cuales, según él, llevan a obtener conclusiones que van de lo observado hacia lo no observado, o sea, a partir de lo ocurrido en nuestra experiencia. También crítica la separación entre datos puros y teoría -criterio con el cual se conduce la tradición positivista-, pues considera que la observación a su vez se encuentra influida por conocimientos teóricos previos; $y$, por lo tanto, ve como imposible justificar lógicamente el principio de la inducción, ni tampoco a través de la experiencia debido a la inexistencia de datos puros, por lo que Popper afirma más bien que el método de las ciencias naturales, es decir el método científico, es enteramente deductivo al tomar en cuenta que este se origina en la existencia de problemas; es decir, no apela a la inducción en ninguno de sus pasos (Popper, 1985, citado por López, 2013). En ese sentido, en cuanto a la inviabilidad de justificar lógicamente el procedimiento inductivo, Dorna (1985) recalca, en un ejemplo sobre la convicción que una persona tenga de "el sol saldrá mañana", que este raciocinio se basa en la experiencia personal, y explica que en este caso se parte de reglas de razonamiento que permiten la predicción del conocimiento, y, por lo tanto, es una petición de principio lógico sobre la uniformidad de la naturaleza. Remarca que "cuando un hecho se repite un cierto número de veces, resulta 
plausible verlo reaparecer" (Dorna, 1985, p. 60). Afirma, de este modo, que desde el inductivismo se tiene como conclusión que existe mayor probabilidad que vuelva ocurrir un hecho que ya venía ocurriendo.

\section{Carga teórica de la observación}

Es de advertir, a su vez, la importancia que tiene el marco de referencia que trae el investigador de la ciencia; de este modo, González (1999) respalda la posición de Popper, al estimar que las hipótesis originales y teorías no se construyen a partir de la observación empírica de datos puros, si no que dicha observación se ve condicionada por las teorías mediante las cuales interpreta el observador. Es decir, coincide con la concepción de Feyerabend, quien afirma que la descripción de un hecho particular es dependiente a una teoría que lo explique; y que el método empírico de la investigación científica permite que haya concordancia entre teorías y hechos; él considera que ninguna teoría antigua debe dejarse de lado, porque puede posibilitar en el futuro el más alto contenido empírico, y llega a explicar de este modo el progreso de la ciencia (González, 1999).

\section{El falsacionismo}

En ese sentido, Popper observa que al inductivismo se oponen tanto las explicaciones lógicas, como las que puedan surgir de la experiencia, y por ello recurre al criterio del falsacionismo. Este será el método propuesto por el autor, dado que mientras no es posible probar deductivamente la verdad de las hipótesis científicas, sí es posible probar su falsedad (López, 2013).

Popper (1980) afirma al respecto que "únicamente decimos que una teoría está falsada si hemos aceptado enunciados básicos que la contradigan [...] aceptamos la falsación solamente si se propone y corrobora una hipótesis empírica de bajo nivel que describa semejante efecto, y podemos denominar a este tipo de hipótesis como hipótesis falsadora" (p. 83).

Popper explica que el método de contrastar rigurosamente las teorías y de seleccionarlas se inicia con el planteamiento de una nueva idea aún no justificada, sea una anticipación, una hipótesis, o teoría, de las cuales se obtienen conclusiones a través de una deducción lógica (Popper, 1980). Sostiene que el requisito elemental que se pide a dicha hipótesis falsadora es que sea empírica para que así pueda ser capaz de refutar lógicamente el enunciado.

\section{Modus tollens}

La manera en que la falsación de una conclusión origina la falsación del sistema total del que se ha deducido, es el modus tollens de la lógica clásica, que se puede explicar de la forma: sea $p$ una conclusión de un sistema $t$ de enunciados, que puede estar compuesto por teorías y condiciones iniciales. Se establece una relación 
de deductibilidad de $p$ a partir de $t$ por medio de $\langle t \rightarrow p »$, que puede leerse: $\langle p$ se sigue de $t\rangle$. Y supongamos que $p$ sea falsa, lo cual puede escribirse $\langle\bar{p}\rangle$ y leerse «no p», que había sido necesario para la deducción del enunciado p, es decir «no $p »$. Dada la relación de deductibilidad, $t \rightarrow p$, y el supuesto $\bar{p}$, podemos inferir $\bar{t}$, es decir, $t$ ha quedado falsado, y a su vez se puede expresar que si p es deducible de $t$ y $p$ es falsa, entonces $t$ es también falso. Utilizando este modo de lógica falsamos el sistema completo, tanto la teoría y las condiciones iniciales (Popper, 1980).

Popper propone la falsabilidad como criterio de demarcación, pero no de sentido, y señala que la falsabilidad separa dos tipos de enunciados: falsables y no falsables (Poper,1980). Y en dicha demarcación de la ciencia no deja de lado la importancia que han tenido las ideas de la metafísica para el desarrollo de la ciencia empírica, a partir de sus aportes creativos (Popper, 1980).

\section{Inducción y fenomenología}

En la historia de la psicología ocurrió un hecho singular, dado que Wundt dejó el laboratorio de psicología experimental en la Universidad de Leipzig (1900) para dedicarse a explorar los fenómenos sociales que tienen su origen en la subjetividad, partiendo desde la conciencia humana y plasmando sus estudios en su obra Psicología de los pueblos; de esta forma, abandona el poder explicativo y predictivo de los estudios experimentales para profundizar más bien en la comprensión de los fenómenos psicológicos (Orbegoso, 2015; Piscoya, 2009b; citados por Sánchez, 2019).

Sánchez emparenta ambos métodos (2019) al proponer que el estudio de los fenómenos sociales, donde se encuentran comprendidos tanto la subjetividad como la intersubjetividad de los individuos, implica asumir uno o más de los modelos propios del enfoque cualitativo, y considera entre ellos al modelo fenomenológico y el inductivista. En nuestro medio existe evidencia de su empleo en investigaciones (Chávez et al., 2017), y también la utilidad de dichos métodos es parte del quehacer profesional en psicología forense.

\section{Hecho y método}

Ambos modelos se nutren epistemológicamente de un determinado hecho que acontece y que es estudiado a través de un método; es así que, tanto Husserl como Strasser, coinciden en que estudiar "un hecho" desde un punto de vista fenomenológico significa otorgarle a la conciencia humana su valor fáctico. El hecho no está sujeto a desarrollo, es decir es algo concretado, el cual se encuentra fuera de nosotros. Y para que podamos hablar de un hecho tenemos que hablar de poder verificar su existencia, y este depende de un método para su aproximación a él. De otro lado, la psicología moderna pretende ser la representante de la observación de la conciencia, pero también dicha posición ya la había asimilado la psicología experimental clásica, que, en su aspiración de convertirse en ciencia natural, elevó 
a la observación científica como legítima fuente de estudio de lo observado. Visto de este modo, tenemos que la psicología experimental tuvo la influencia del romanticismo alemán y su concepción de la naturaleza como unidad viva, observándose similitudes entre sus postulados al enfocar problemáticas tanto científico-naturales como filosóficas y psicológicas (García, 1979).

Particularmente, el hecho es la lectura de una experiencia, pero también requiere una interpretación a través de un método o teoría; porque en el histórico caso de la caída de la manzana sería diferente la interpretación si el observador lo fuera Adán o Newton (Piaget, 1968, citado por García, 1979). Tal cual ya se propuso la relación entre hecho y método, según Strasser (1967) explica, por ejemplo, que para los psicólogos estudiar la inteligencia depende de aplicar un método aceptado dentro de la comunidad científica, es decir, basarse en lo que un test determinado mide. Por ende, Strasser aclara que los científicos sólo verificarán un hecho si se encuentran en la misma línea metódica que lo explique, y aclara que, en el caso de Freud y Adler, trataban la misma clase de clientela en igual época, pero nunca sucedió que Freud diagnosticara un sentimiento de inferioridad, o que Adler observara un complejo de Edipo. Los hechos eran diferentes dado que el enfoque metodológico también lo era.

\section{Inducción}

Según el inductivismo, el conocimiento científico se inicia con la observación; a su vez, los enunciados observacionales permiten establecer leyes y teorías. Los enunciados observacionales son del tipo "Juan empujó a su hermano", y son singulares porque proceden de la observación que hace el científico en un lugar y tiempo determinado. De otro lado, los enunciados generales o universales son aseveraciones sobre las propiedades o la conducta de algún aspecto de la naturaleza; por ejemplo, que todos los animales tienen necesidad innata de descargar impulsos agresivos, lo cual corresponde por tanto a una ley o teoría. De este modo, los inductivistas consideran que es válido generalizar, desde de una cantidad finita de hechos observados, a una ley determinada; pudiéndose decir que el inductivismo se basa en el siguiente principio: si en una numerosa variedad de condiciones se observa una elevada cantidad de $\mathrm{X}$, y si todas las $\mathrm{X}$ observadas tienen la propiedad Y, entonces todas las X tienen la propiedad Y (Ramírez, 2014).

Para esto, Husserl explica que en este mundo donde cohabitan tanto el investigador de la naturaleza como el ser humano lego, este último se plantea preguntas sobre el mundo, las cuales pueden ser respondidas desde leyes científicas que se confirman en la experiencia, mediante observaciones y experimentos que corresponden al terreno de las inducciones (Husserl, 2008). También acota que la evolución que tuvo la geometría hacia la física se observa desde los antiguos pitagóricos, a quienes les impresionaba, por ejemplo, la dependencia funcional en que se producía el sonido respecto a la longitud de una cuerda puesta en vibración; 
además, que en esos tiempos se tenía ya conocimiento de otras causalidades similares en materia de conocimiento. Es decir, había dependencia de los fenómenos y las formas; pero, en general, faltaba una mejor explicación de la trama de las dependencias causales; o sea, fue la medición de los contenidos de dichas formas lo que inspiró a Galileo y significó para él encontrar en la física el conocimiento certero (Husserl, 2008).

De otro lado, Husserl destaca el extraordinario mérito que hay que otorgarle también a Brentano, quien, en su intento de reforma de la psicología, indagó en las vivencias de la conciencia, concluyendo que tienen un carácter intencional. Plantea también aceptar la vida de la conciencia donde se encuentran datos validos acerca de los colores, los tonos, entre otras características del objeto, y donde se alojan datos sobre la experiencia sensible, y para darle credibilidad lo ejemplifica del siguiente modo: "el 'yo veo un árbol que es verde, yo oigo el murmullo de sus hojas, yo huelo sus flores', etc.; o 'yo me recuerdo en mis años de escuela', 'yo estoy afligido por la enfermedad de mi amigo', etc.” (Husserl, 2008, p. 271).

\section{Fenomenología}

Para Husserl, los físicos de la experiencia física, los biólogos de la experiencia biológica, así como los científicos de la experiencia de las ciencias del espíritu, todos ellos, se llaman legítimamente ciencias de la experiencia, porque recurren a ella. Admite que, en la experiencia humana, el hombre está seguro de la realidad efectiva de las cosas reales de las que él se ocupa, pero a su vez admite que también hay un lado "inconsciente", y que aquí se encuentran incluidos los afectos reprimidos del amor, de la animadversión, así como las motivaciones inconscientes, los cuales también tienen una validez intrínseca (Husserl, 2008).

En ese sentido, para llegar al punto central de la "psicología descriptiva" propia de los fenómenos que se estudien, es necesario un método que Husserl denomina: "reducción psicológico-fenomenológica". Y desde el lugar de la práctica de una psicología científica empírica, él tiene claro que tiene objetividad con las intencionalidades del percibir humano, y expresa: "que sea ser o ilusión no cambia nada en el sujeto concerniente que lleva a cabo, por ejemplo, una percepción, de hecho la conciencia tiene 'este árbol allí', que lleva a cabo en eso aquella simple certeza que pertenece a la esencia del percibir...” (Husserl, 2008, p. 274). Así mismo, Husserl expresa que cuando se trata de la percepción de una persona, el psicólogo se abstiene de la coverificación de la validez que lleva a cabo la persona que percibe. Asimismo, el psicólogo lleva dentro un "observador desinteresado" e investigador de sí mismo como de otros, quien, dentro de su investigación, no debe ni tomar parte de las validaciones de las personas que para él son su tema de consulta. A su vez, señala que el psicólogo no quiere sino lo mismo que las ciencias positivas, a través del método de la reducción fenomenológica, o sea, alcanzar el verdadero autoconocimiento en la persona (Husserl, 2008). 


\section{Perspectiva fenomenológica en violencia}

Dentro de los diferentes matices que conciernen al estudio fenomenológico de la violencia, Benyakar (2003) diferencia tres aspectos importantes cuando ocurre una exposición a situaciones violentas: a) El evento fáctico, que alude a la situación de violencia que tiene el poder de alterar su homeostasis o equilibrio; agrega el autor que dicho efecto se agudiza al ser inesperado, y si presenta elementos novedosos que pueden ser poco comprensibles para la persona padeciente, los cuales puedan amenazar la integridad física y psicológica, afectando la autoconfianza y el vivir diario; b) la vivencia, que es el sentimiento que suscita la violencia; a su vez, la vivencia traumática tiene el poder de no permitir asimilar a través del pensamiento lo ocurrido, contiene procesos que pueden no ser del todo conscientes; c) la experiencia, que es la capacidad que tiene la persona para metabolizar los sentimientos que resultan de la violencia; es pensable y comunicable, es la percepción que se tiene de los efectos del hecho ocurrido.

En ese sentido, la intervención profesional intelectiva y comprensiva con personas en violencia implica manejar con ellos ciertos niveles de acercamiento, a lo que Husserl se refiere no como certezas sino como "intuiciones significativas" sobre la realidad que vienen atravesando en una determinada coordenada espacio-temporal, que se confirma en el mundo de la experiencia. Interesa no solo la explicación racional del porqué de los hechos que acaecen en la víctima, sino también su mundo vital captado desde lo afectivo y volitivo. Desde una perspectiva fenomenológico-existencial, se valoriza ese mundo circundante de la víctima que Husserl validaba de espontaneo, y ante el cual, como científicos, habría que no contaminarnos con prejuicios tanto personales como sociales, para aplicar una epojé (suspensión, en griego) o reducción fenomenológica, o sea, detener nuestros juicios críticos para centrarnos en las intencionalidades de la víctima, propios de su conciencia (Martínez-Romero, 2018).

De este modo, Pavón (2020) se pregunta: ¿qué necesidad tuvo Husserl en su fenomenología para llegar a este nivel de reflexión?, llegando a la conclusión que fue debido a que las ciencias naturales; según Husserl, estaban cargadas de un realismo ingenuo que tomaba la realidad como separada de la conciencia. Y en ese sentido comparte con él la idea de que la epojé es una exigencia a todas las ciencias objetivas, y, de este modo, la epojé de las ciencias objetivas permite identificar aquellos conocimientos incuestionables de cada ciencia. Asimismo, puso en primer plano el mundo de la vida y a no pasarlo por alto, es decir, Husserl nos invita a cuestionar lo ya sabido y conocido de las ciencias objetivas. Está claro que las víctimas enfrentadas a la violencia sufren emociones negativas, y al respecto Husserl tomó en cuenta que "el mundo de la vida" es una vivencia fidedigna, y representa que lo experimentado es mucho más significativo que, por ejemplo, lo vertido en una entrevista, que a su vez son vivencias que la ciencia natural deja de lado cuando la víctima es atendida por sus lesiones físicas por médicos y forenses, 
que prestan mayor interés a dichos hechos que a la vivencia interna de la consultante. El paso de convertir "lo humano" en fenómeno, al margen de la magnitud del hecho, implica entender que los hechos ocurridos impresionan en la conciencia de la víctima, $y$, por ende, son emociones de valor incuestionable dentro del marco de la psicoterapia (Martínez-Romero, 2018).

$\mathrm{Y}$ es de mencionar que una de las bases del desarrollo de la psicología del siglo XX fue la observación acuciosa del pasado individual; sin embargo, desde la psicología fenomenológica existencial se considera que en la vida lo más importante se encuentra en el camino por recorrer, y, por tanto, una vida con sentido implica desarrollar un proyecto futuro donde se deben superar las frustraciones del proceso de violencia vivido (Längle, 2013; citado por Martínez-Romero, 2018). De este modo, la tarea profesional que desempeñamos como psicólogos, desde la perspectiva fenomenológico-existencial, tiene como objetivo llevar a la conciencia de la persona maltratada el hecho de que ha sido coartado su sentido de libertad (Martínez-Romero, 2018).

Otro aporte del enfoque fenomenológico sobre las vivencias de las mujeres adultas víctimas de violencia conyugal, lo brinda la investigadora Gil (2018), quien puntualiza que, en un inicio, mediante el método de reducción fenomenológica, se identifica la percepción y el significado de los discursos de las víctimas para comprender lo esencial del fenómeno de cómo viven o sienten. Luego, dichos discursos se clasifican en unidades de significado a la luz de los conceptos de Heidegger y de su obra Ser y Tiempo, para así descubrir el "ser-ahí" que se encuentra al interior de las vivencias de las víctimas. Y, a través del trabajo interpretativo, se van develando premisas importantes, tales como que el hecho de ser mujer y a la vez llevar el estigma de víctima en nuestro medio, es una condición penosa y con una carga de angustia y carácter de trauma en dicha experiencia de vida, más aún al tener que compartir opiniones y percepciones del agresor y así anularse ella misma; de este modo, ella imagina el rechazo social por su condición de víctima, y explica Heidegger que dicha situación angustiante la lleva a aceptar la ayuda profesional para liberarse del maltrato y del agresor.

Paz-Gonzales y Pino-Ramírez (2011) sostienen que el enfoque epistemológico empírico-inductivo bien representa al paradigma positivista, y que sus hallazgos surgen del contacto que la persona tiene con la realidad mediante el uso de los sentidos; y que en dichas investigaciones se busca apreciar la realidad basándose en la frecuencia con que ocurren los hechos, siendo ésta la fase inicial para comprender la naturaleza de la problemática de la violencia familiar.

\section{Método hipotético deductivo}

Popper (1980) considera que dar una explicación causal de un acontecimiento es obtener una conclusión que resulta de la interacción de leyes universales y 
enunciados singulares (condiciones iniciales). Por ejemplo, cuando ocurre una rotura de un trozo de hilo, y tenemos la información que éste tenía una resistencia a la fuerza de libra $1 x$, al que se había ejercido un peso de $2 x$ libras; nos encontramos que dicho acontecimiento está compuesto por los 2 elementos antes mencionados, y surge así la hipótesis: «Siempre que se cargue un hilo con un peso mayor al que caracteriza la resistencia a la tracción del mismo, se romperá»». De este modo, tenemos: 1) enunciados universales, o hipótesis que tienen el carácter de leyes naturales, y 2) enunciados singulares, que se aplican al acontecimiento ocurrido. Se puede decir que las condiciones iniciales son la causa del acontecimiento (en este caso, la causa de que se rompa el hilo fue que se había ejercido una carga de $2 x$ libras a un hilo que tenía una resistencia a la tracción de $1 x$ libra); y la predicción de que se rompiera el hilo sería el efecto. Podemos observar así la aplicabilidad universal del método deductivo.

Dicha apreciación de Popper nos da luces del nivel del alcance y exigencia que tiene toda ley científica; es por ello que en la vertiente del enfoque cuantitativo aplicado a la psicología se espera, por tanto, acercarnos en lo posible a mediciones cada vez más fidedignas de sus variables.

\section{El enfoque cuantitativo en psicología}

Según Sánchez (2019), el trabajo de realizar una investigación científica obliga a decidirse por el uso de dos tipos de enfoques: el cualitativo y el cuantitativo. Sánchez puntualiza que el enfoque cuantitativo fue ideado para estudiar fenómenos que ocurrían en las ciencias naturales, y que los conocimientos que se desprendieron de dichas disciplinas fueron también de utilidad en la medicina, y sus ramas aplicativas; así como en la informática extendiéndose incluso a las telecomunicaciones. Dicho enfoque tiene sus orígenes en el empirismo inglés, el positivismo francés y el neopositivismo alemán; y tiene dos modelos aplicativos: el modelo hipotético deductivo y el causal explicativo.

\subsection{El modelo hipotético-deductivo}

Se basa en la creación de hipótesis a partir de dos proposiciones, una universal (leyes y teorías científicas) y otra empírica (el fenómeno en estudio), para someterla luego a contrastación (Sánchez, 2019). Este modelo tiene la finalidad de explicar y predecir los fenómenos y se emplea en todas las ciencias empíricas, apoyándose en la medición y en los hechos de la experiencia; su meta es la obtención de leyes científicas.

En la terapéutica psicológica dicho enfoque se emplea cuando, por ejemplo, el objetivo fuera averiguar la cronicidad de los síntomas que tiene alguien que se presume tiene un cuadro depresivo mayor, y en base a esta hipótesis -utilizando el modelo hipotético-deductivo- bastaría aplicarle un test psicológico confiable y valido para medir la magnitud del problema y luego de llevar a cabo una entrevista 
estructurada con la persona en varias sesiones, y bajo la observación de sus síntomas, ubicar la nosología dentro de un manual de psicodiagnóstico y/o en algún respaldo teórico que sustente la existencia de dicho padecimiento, y así estructurar el tratamiento basado en una determinada corriente terapéutica, y, finalmente, aplicarlo considerando los parámetros de tiempo de recuperación que reportan los estudios experimentales en dicha casuística, tal cual un médico procede al abordar una enfermedad viral o bacterial en un paciente (Sánchez, 2019). Según este modelo, luego de formular hipótesis en base al bagaje teórico y a los hechos que se observan ocurren en la problemática en estudio, estas hipótesis son llevadas a su contrastación empírica a través de pruebas e instrumentos fidedignos, y luego se analizan los resultados que deben ser expuestos para su puesta a juicio y réplica por la comunidad científica, de manera tal que se pueda evaluar su objetividad (Piscoya, 2009a, 2009b, citado por Sánchez, 2019).

El desarrollo del modelo hipotético-deductivo comprende tres fases (Ballesteros y García, 1995b; Fernández-Trespalacios, 1986b; Llor et al., 1995b; Grzib y Briales, 1996b; citados por Ortego et al., 2011):

\section{Observación}

Es la fase de descubrimiento del problema que se va a investigar, parte de la presencia de una duda o problema por investigar, aunque también puede ser accidental, tal es el caso de Pávlov, que, estudiando la fisiología de la digestión en los perros, se encontró con el fenómeno de los reflejos condicionados. Sea la observación de tipo accidental o sistemática que se hace del fenómeno, esta requiere dos requisitos para que tenga el carácter de científico: primero tiene que registrar un fenómeno que pueda ser susceptible de ser medible; $y$, en segundo lugar, tiene que tratarse de un acontecimiento que se pueda repetir, ya que para poder aceptar o rechazar la hipótesis respecto a dicho fenómeno es necesario poder replicar el fenómeno que se está estudiando (Ortego et al., 2011).

\section{Formulación de hipótesis generales que expliquen los hechos acontecidos}

En relación al concepto de hipótesis tenemos que: "las hipótesis indican lo que tratamos de probar y se definen como explicaciones tentativas del fenómeno investigado, se derivan de la teoría existente...” (Hernández et al., 2014, p. 104). Es decir, una hipótesis es una suposición que realiza el investigador en forma de enunciado, cuya principal característica es que puede ser sometida a contrastación experimental. Los enunciados de las hipótesis siguen generalmente la estructura "si... entonces" y detallan bajo qué condiciones se espera que ocurra un resultado o resultados. En la medida que más datos particulares deducidos de la hipótesis no sean rechazados por la experimentación, la probabilidad de la hipótesis se eleva, sin embargo, bajo ninguna circunstancia la certeza es algo definitivo porque puede surgir una observación que refute la hipótesis, lo cual 
es una característica particular de las ciencias empíricas que siempre son probabilísticas (Ortego et al., 2011).

El científico a partir de esas observaciones plantea una hipótesis general, sin embargo, esta no puede ser sometida a la verificación experimental por lo que el científico tiene que deducir de la hipótesis general un caso concreto que pueda ser comprobado con los datos empíricos; es decir, formula subhipótesis a partir de la hipótesis general (Ortego et al., 2011).

\section{Verificación o contrastación de la hipótesis}

Para concluir si la hipótesis debe ser aceptada o rechazada, el investigador utiliza dos métodos: experimentales y no experimentales; la diferencia principal entre ambos métodos consiste en el grado de control que se acciona sobre el fenómeno estudiado (Ballesteros y García, 1995b; Fernández-Trespalacios, 1986b; Llor et al., 1995b; Grzib y Briales, 1996b; citados por Ortego et al., 2011).

\section{a) Métodos no experimentales}

- En la observación sistemática el investigador registra y mide los fenómenos tal y como se producen en su ambiente natural, sin manipular las causas de forma intencional, el investigador no controla ninguna de las posibles variables extrañas que pueden influir en los resultados. Un ejemplo de ello lo tenemos en Darwin, quien desarrolló la teoría evolucionista a partir de la observación de diferentes especies animales en la adaptación a su medio.

En los casos de violencia, desde un plano observacional, existen indicadores de afectación que se desprenden de la sola observancia de los síntomas de la realidad conflictiva de la víctima. En ese sentido, Lincoln y Guba (1981, 1982, 1985, 1986, citados por Arias y Giraldo, 2011), proponen criterios de rigor para considerar los hallazgos de una investigación como conocimiento científico. Y siguiendo la misma línea, se plantea la analogía que existe entre el criterio de confirmabilidad (investigaciones cualitativas) y el de objetividad (investigaciones cuantitativas) ambos referidos a: "la neutralidad en el análisis y la interpretación de la información, que se logra cuando otros investigadores pueden seguir la pista y llegar a hallazgos similares" (Castillo y Vásquez, 2003, citados por Arias y Giraldo, 2011, p. 503); es decir, un caso de violencia como tal, puede ser validado también por otro profesional basándose ambos en criterios científico observacionales.

- El método correlacional consiste en aplicar el análisis estadístico a datos previamente clasificados, para determinar si están relacionados o no; siendo que aquí se desconoce cuál de los acontecimientos se comporta 
como causa o efecto, pues el objetivo que pretende es precisar el grado y la dirección de la correlación. Y en el estudio de los casos de violencia, se puede afirmar que la persona evaluada tiene alta comorbilidad con casos similares de personas víctimas de violencia, presentando diferentes síntomas, según confirman los sustentos empíricos de las investigaciones, tal como se ha encontrado, por ejemplo, en la relación entre victimización por violencia de pareja y puntajes altos en la prueba del MMPI en escalas como: hostilidad, malestar social, problemas de pensamiento y concentración, así como bajas puntuaciones en defensas psicológica entre otros; además estos estudios también han hallado una correlación entre las puntuaciones del MMPI y la magnitud de la violencia de la pareja tanto física como psicológica (Khan et al., 1993; Rollstin y Kern, 1998; citados por Torres, 2014).

\section{b) Métodos experimentales}

Permiten establecer relaciones de tipo causa-efecto; el experimento es llevado a cabo para poner a prueba la hipótesis sobre las relaciones existente entre dos tipos de variables. A la variable que manipula el experimentador se le conoce como variable independiente, mientras que la variable conductual que estamos interesados en medir es la variable dependiente. En este caso, se tiene que controlar las variables extrañas que puedan afectar los resultados del experimento (Ortego et al., 2011).

\subsection{El modelo causal-explicativo}

Se sostiene en la puesta a prueba de hipótesis causales y en situaciones controladas de laboratorio (control de las variables extrañas). Tiene relación con el modelo hipotético-deductivo, pues las hipótesis se enuncian al modo lógico de "si p, entonces q"; o sea, "si se manipula tal causa, entonces ocurrirá tal consecuencia", o "si se suministra tal reactivo a tal compuesto, entonces se obtendrá tal consecuencia". Fue desarrollada por Hempel (Okasha, 2002), quien aceptó las limitaciones de tal modelo, dado que no todos los fenómenos de la realidad y sobre todo los sociales se aproximan a las explicaciones causales de este modelo (Sánchez, 2019).

En ese mismo sentido, Creswell y Reichardt (citados por Hernández et al., 2014) denominan a los experimentos estudios de intervención, porque el investigador en algunos casos reproduce una situación para tratar de explicar cómo esta afecta a las personas expuestas a dicho evento, en comparación con quienes no lo estén; por ejemplo, en niños expuestos a programas de televisión con contenido de conducta antisociales habrá una mayor tendencia a una elevación de conductas violentas que aquellos que no lo estén. Este modelo explica que los experimentos se caracterizan por manipular tratamientos, variables psicosociales, o intervenciones (variables independientes) para causar efectos en otras variables (dependientes), 
manteniendo el control de otros factores que puedan interferir en los resultados, o también llamada validez interna (Hernández et al, 2014).

En este sentido, se aprecia que el planteamiento a nivel causal-explicativo de las investigaciones sobre violencia familiar, exige, pues, plantear hipótesis causales o de diferencia de grupos (Hernández et al, 2014), donde se comparen: un grupo de personas sin violencia y un grupo de personas víctimas de violencia, medidos en una variable dependiente, para así confirmar los efectos de la violencia, donde la hipótesis que surge, en base a teorías cognitivas existentes sobre los efectos de la violencia, sería: "la violencia ocasiona afectación psicológica en las personas"; sin embargo, para lograr un mejor ajuste y poderse verificar experimentalmente -dado que el carácter general de tal hipótesis no es deducible de la misma- se formulan subhipótesis específicas que se encuentran operacionalizadas (es decir, que son plausibles de medición). De este modo, al observar que la hipótesis planteada es muy genérica, para que pueda ser pasible de contrastación empírica se proponen nuevas hipótesis más específicas (Hernández et. al 2014); por ejemplo: "si determinados sujetos se exponen a eventos de violencia (física y/o psicológica), entonces desarrollarán mayores niveles de frustración, depresión, baja autoestima, estrés, desesperanza aprendida, soledad (entre otras consecuencias) que aquellos sujetos que no se expongan a dichos eventos".

En dicho sentido, la terapia cognitiva apoya la perspectiva planteada líneas arriba, al explicar que la percepción de los hechos es la que predice las emociones, la conducta de un individuo, e incluso su sintomatología (Beck, 1967, 1976; citado por Jaramillo, 2011). También es de utilidad el concepto de creencias de Ellis (1989, 1990, citado por Jaramillo, 2011), quien las cataloga como moldes cognitivos mediante los cuales evaluamos de forma idiosincrática nuestra experiencia.

De este modo, debe entenderse que la elaboración cognitiva mediatiza el impacto del hecho violento llevando a adoptar una respuesta minimizante o maximizante de sus consecuencias. A lo cual ya Ellis, en 1955, expresaría que "el hombre es un indigente racional que con facilidad adquiere ideas disfuncionales que lo llevan a forjar distintos trastornos emocionales" (Aquino y Rodríguez, 2019).

A su vez, la contribución de las investigaciones de Beck (1962) en el marco de la terapia cognitiva facilita a los investigadores venideros explicar tanto el pensamiento racional como el desadaptativo, valiéndose de técnicas psicológicas para que el individuo se adapte exitosamente a su medio (Caro, 2013, citado en Aquino y Rodríguez, 2019).

Finalmente, en este acercamiento a dicha complejidad de manifestaciones psicológicas provocada por las experiencias de violencia, se puede decir que, tras desencadenarse los trastornos emocionales y adaptativos, existe, con el empleo 
de la terapia cognitiva, la posibilidad de poder convertirse en personas fuertes y emocionalmente estables (Santandreu, 2014).

\section{Vínculos de la inducción, deducción y fenomenología con estudios de la casuística en violencia familiar}

Existen disponibles varios estudios de enfoques cualitativo y cuantitativo, que aportan con resultados significativos respecto a la casuística en violencia familiar. En cuanto a los trabajos cualitativos, se considera, en primer lugar, el de Nóblega y Muñoz (2009), que realizaron su estudio en un asentamiento humano del distrito de Villa El Salvador. A través de grupos focales, se exploraron los objetivos del estudio, que consistieron en describir las características de la violencia hacia la mujer, identificar las creencias que las mantienen unidas al agresor, y de describir los factores que frenan la violencia, todo ello desde la perspectiva del modelo ecológico. Se utilizó como instrumento una guía de grupo focal. Los principales hallazgos se centran en que los rasgos de déficit de autoconfianza y estima propia son los que predominan en las mujeres informantes, así como una percepción del agresor como un sujeto machista, desconfiado y con conductas agresivas, las cuales son características del nivel individual del modelo ecológico y que son, a su vez, un factor de riesgo. Así mismo, se evidenció que el consumo de alcohol y los conflictos con relación a la crianza de los hijos, se presentan como disparadores de la violencia en la pareja. También las entrevistadas reconocen la importancia que ellas otorgan a su rol activo para evitar los conflictos. De otro lado las autoras aseveran observar factores atenuantes en la manifestación de los actos de violencia familiar, dado que las mujeres participan más del mercado laboral, lo cual reduce efectivamente la presencia de la violencia, al existir igualdad de roles entre el hombre y la mujer; en consecuencia, tales factores atenuantes conformarían un factor protector del exosistema para ser menos expuestas a situaciones de agresión. Con relación a los factores que mantienen a la mujer unida al agresor, uno de los factores de riesgo a nivel individual viene a ser la vivencia de temor que impide a la mujer agredida reaccionar y actuar en función a la contingencia de agresión. Y a nivel de macrosistema, la sobrevaloración que tienen de su rol materno las mujeres violentadas constituye un factor que incide en su pasividad para no separarse.

También se dispone del trabajo de Cueva et al. (2016), quienes emplearon la metodología cualitativa a través del análisis de las cartas del proyecto "Cartas de Mujeres. Voces que rompen el silencio de la violencia" (cartas que provenían de Lima y otras regiones del Perú), y también de entrevistas a profundidad (de mujeres que vivían en Lima), información reunida a partir de la cual se analizaron las subjetividades de las mujeres que han sufrido violencia psicológica por parte de sus parejas, el afronte que emplean en algunos casos para poder dejar la relación, así como los efectos de la violencia. Se concluyó que la violencia psicológica por parte de sus parejas pretende disminuir su autoconfianza y generarles dependencia; como también que las mujeres han tolerado la violencia psicológica debido a 
mandatos sociales de género patriarcales que ellas asumen como propios; a la vez que se han adjudicado roles de cuidado y de compromiso frente a su prole y a sus parejas, a pesar del contexto de violencia en el que viven. Entre los afrontes para dejar la relación violenta, se encontró que las mujeres que lo lograron lo hicieron a través de grandes modificaciones en sus hábitos y estilos de vida, y/o debido a alguna desavenencia impactante; también lo lograron mediante la búsqueda de ayuda de terceras personas o por la intervención de entidades de ayuda pública; por último, entre los efectos negativos de la violencia se encontraron sentimientos como tristeza. impotencia, miedo, resignación y ansiedad.

Otro estudio es el de Vásquez et al. (2016), enfocado en la investigación cualitativa interpretativa, cuyo propósito fue analizar el significado de la violencia conyugal y su impacto en la vida de mujeres del Municipio de Cajicá, Cundinamarca, Colombia. Se realizaron análisis de contenido de las entrevistas en profundidad y se abarcaron temas como: a) familia-relación conyugal, b) paternidad y crianza, c) derechos humanos, d) comunicación y e) empoderamiento.

Los resultados más importantes indican que la violencia conyugal es un fenómeno multidimensional que afecta las diferentes áreas de la vida de las víctimas, así como de su entorno, lo cual se evidencia en las categorías negativas de: sufrimiento, cansancio, sumisión y miedo, como indicadores de la pérdida del logro de ideales en la víctima. Se encontró, asimismo, un desgaste emocional en ellas, tratando de hacer reacomodos a la relación, afectándose también su rol de madre. A partir del análisis de contenido, sobresale un aspecto referido al poder de los factores socioculturales (la religión y las costumbres) en el mantenimiento de la relación conyugal. En este caso, la fe en la religión se manifiesta como coadyuvante en el sostenimiento de la relación; a su vez, las costumbres culturales intervienen como elementos justificantes de la persistencia de la violencia conyugal. Las categorías que las muestran con una visión positiva son: lucha, sacrificio, perdón, respeto, amor, comprensión y determinación. Cada una de estas categorías propician un núcleo de significados para así poder sobrellevar los aspectos negativos de la relación conyugal, y de esa manera, poder brindar afecto a la familia.

Concerniente a los trabajos cuantitativos, se cuenta en nuestro medio con el estudio de Huerta et al. (2014), que investigaron sobre los efectos de la violencia en ansiedad y depresión según las características de convivencia, en zonas urbanomarginales de la ciudad de Lima, en una muestra de 428 mujeres; el diseño fue descriptivo, correlacional y transversal; se encontró una correlación significativa entre la ansiedad estado y la depresión, y una correlación muy significativa entre depresión y ansiedad rasgo; así mismo, se observó que las mujeres víctimas de violencia tuvieron puntajes más altos de depresión que las no violentadas.

Por su parte, González et al. (2020) realizaron un estudio descriptivo y transversal en 5 colegios estatales del distrito de Puente Piedra, con el objetivo 
de determinar la frecuencia de la violencia de pareja, para lo cual emplearon la versión española del Cuestionario de Conflictos en las Relaciones de Pareja en Adolescentes (CADRI), aplicándose encuestas individuales auto administradas a una muestra de 208 adolescentes, que en su mayoría cursaban el tercero y cuarto de secundaria. La variable violencia y sus componentes fueron agrupados en violencia cometida y violencia sufrida, y para el análisis bivariado de las variables se utilizó el estadístico chi cuadrado, considerando los valores con nivel de $\mathrm{p}<0,05$ como significativos. Se estimó, además, el odds ratio (OR) con intervalo de confianza al 95\%. Entre los resultados se encontró que la violencia cometida y la violencia sufrida se presentaron en ambos sexos; la violencia cometida tuvo valores más altos en los varones, sobre todo en el componente relacional y amenaza; la violencia sufrida se presentó con valores más altos en los varones y ocurrió principalmente en el componente verbal emocional, amenaza y violencia física. Estos resultados llevan a concluir que la dinámica de la violencia en las parejas adolescentes está signada básicamente por el sexismo.

El trabajo de Colque (2020) tuvo el propósito de determinar los efectos psicológicos en una muestra de 108 mujeres denunciantes de violencia por parte de su pareja, en la provincia constitucional del Callao. El diseño utilizado fue el no experimental, transversal y de alcance descriptivo. Se aplicó la Lista de Chequeo de Síntomas de Distrés SCL-90 R de Derogatis. Como resultado se encontró que los síntomas psicológicos de las mujeres víctimas son superiores a la población psiquiátrica ambulatoria, basándose en la cantidad promedio de síntomas positivos hallados; así, los síntomas con promedios más altos fueron: depresión, obsesióncompulsión, ansiedad y somatización.

\section{CONCLUSIONES}

En la investigación en psicología, tanto la fenomenología, como la inducción y la deducción, tienen su origen epistemológico en la experiencia. Y también de forma circular, las experiencias son explicables desde teorías que se contrastan y de las que se deducen dichas experiencias.

Ni la verificación ni la falsación son definitivamente concluyentes, y cuando los hallazgos de nuevas investigaciones, al ser refutados o verificados, no aportan nada nuevo, un investigador puede dar un giro descriptivo y explicativo hacia la corriente fenomenológica.

La investigación cualitativa no tiene por finalidad contrastar hipótesis para generar leyes y teorías con la precisión y relación de causalidad del enfoque cuantitativo, pero los conocimientos que de ellas emanan son parte del acervo científico, y a la vez de utilidad para la ciencia inductiva y deductiva, aunque limitado al contexto social e histórico-cultural en el que se desarrolla. 
Desde el paradigma positivista, neopositivista y falsacionista actual de la ciencia, el avance científico se obtiene cuando falseamos una hipótesis qua ya no tiene capacidad explicativa y que exige que el investigador se apoye en hipótesis mejores.

Como toda investigación, los estudios en violencia también se enmarcan dentro de una convención de académicos que definen operacionalmente los términos que se investigan, que a su vez responden a la corriente psicológica explicativa que se elija.

Siendo muy escasas las investigaciones de corte experimental y longitudinal en violencia familiar, se espera poder contar con la reglamentación administrativa, protocolar, ética y curricular indispensable, a nivel institucional universitario o del aporte privado, que facilite, de ese modo, su implementación, despliegue y desarrollo en nuestro contexto sociocultural.

Las personas que padecen violencia, además de constituir un terreno de estudio científico mediante el método hipotético-deductivo, también permite la incursión del diseño fenomenológico al narrar dichas personas su historia de vida.

\section{Fuentes de financiamiento}

La investigación fue autofinanciada por el propio autor.

\section{Conflicto de intereses}

El autor declara no tener ningún conflicto de interés económico, institucional, laboral o personal al realizar el manuscrito.

\section{REFERENCIAS}

Aquino, F. y Rodríguez, J. (2019). Distorsiones cognitivas y dependencia emocional en miembros de la comunidad LGTB víctimas de violencia de pareja [Tesis de licenciatura, Universidad Nacional de San Agustín de Arequipa]. Repositorio institucional de la Universidad Nacional de San Agustín de Arequipa. http://repositorio.unsa.edu. pe/bitstream/handle/UNSA/10318/PSaqcafy.pdf?sequence=1\&isAllowed=y

Arias, M. y Giraldo, C. (2011). El rigor científico en la investigación cualitativa. Investigación y Educación en Enfermería, 29(3), 500-514. https://www.redalyc.org/ pdf/1052/105222406020.pdf

Barceló, E. (2016). La ciencia de Hildegarda. https://www.academia.edu/27538733/La_ ciencia_de_Hildegarda

Benyakar, M. (2016). Lo disruptivo y lo traumático: Abordajes posibles frente a situaciones de crisis individuales y colectivas. Universitaria. 
Caro, I. (2013). El Estudio de la Personalidad en el Modelo Cognitivo de Beck. Reflexiones Críticas. Departamento de Personalidad, Evaluación y Tratamientos Psicológicos de la Universidad de Valencia.

Chávez A., Orihuela A., Rodríguez S., Villanueva, M., Quispe, J. (2017). Caracterización de la investigación sobre violencia familiar en Universidades de Lima Metropolitana. Cátedra Villarreal Psicología, 2(2), 371-386. http://revistas.unfv.edu.pe/index. php/CVFP/article/view/323/289

Colque, J. (2020). Consecuencias psicológicas en mujeres víctimas de violencia de pareja. Educa UMCH. Revista sobre Educación y Sociedad, 2020, 15(1), 5-22. https://doi. org/10.35756/educaumch.vli15.129

Cueva, S., Mendiola F. y Valega C. (2016). Subjetividades y violencia: Una aproximación a las subjetividades de mujeres que han sufrido violencia psicológica por parte de sus parejas. En "Voces que rompen el silencio de la violencia. Concurso de Investigación sobre violencia de género". Editado por Pontificia Universidad Católica del Perú. https://www.mujereslibresdeviolencia.usmp.edu.pe/wp-content/uploads/2017/03/ DARS GIZWEB.pdf

Dávila, G. (2006). El razonamiento inductivo y deductivo dentro del proceso investigativo en ciencias experimentales y sociales. Revista Laurus, 12(Ext), 180-205. https:// www.redalyc.org/articulo.oa? $\mathrm{id}=761 / 76109911$

Dorna, A. (1985). ¿Deductivismo versus inductivismo? Croquis para una reflexión tolerante en psicología. Revista Latinoamericana de Psicología, 17(1), 57-71. https:// www.redalyc.org/articulo.oa?id=805/80517102

Echevarría, J. (1999). Introducción a la metodología de la ciencia: la filosofía en el siglo $X X$. Cátedra.

García, L. (1979). Instauración y crisis de la observación en la Psicología moderna. Revista de Psicología, 7. http://www.memoria.fahce.unlp.edu.ar/art_revistas/pr.2430/ pr.2430.pdf

Gil, M., Schillagi M., Squadrito, S. y Escalante E. (2011). Evaluación y análisis fenomenológico del relato en pericias realizadas por abuso sexual en niños/as-adolescentes. Liberabit, 17(2), 157-171. http://www.scielo.org.pe/scielo.php?script=sci_arttext\&pid $=\mathrm{S} 1729-48272011000200006$

Gil, Y. (2018). Viviendo la violencia conyugal: un enfoque fenomenológico [Tesis de licenciatura, Universidad Nacional de Trujillo]. Repositorio institucional de la Universidad Nacional de Trujillo. https:/dspace.unitru.edu.pe/bitstream/handle/UNITRU/13742/1827.pdf?sequence=1\&isAllowed $=y$

Gonzales, A. (2006). Del utillaje conceptual de la antropología: los usos del término "inductivismo" y los usos del término "hermenéutica". Dos propuestas de clarificación. Revista de Antropología Social, 15, 327-372. https://dialnet.unirioja.es/servlet/ articulo?codigo $=2545281$

Gonzalez C., Cervera W., Alvarado V., Izquierdo A., Neyra W., Morales J. (2020). Violencia de pareja en adolescentes estudiantes de colegios estatales de Lima Norte. Rev. 
Peru. Investig. Salud; 4(2): 57-64. http://revistas.unheval.edu.pe/index.php/repis/article/view/330/573

González, R. (1999). Percepción - Teorías: desde el positivismo lógico hacia la ciencia cognitiva [Tesis de maestría, Universidad de Chile]. Repositorio institucional de la Universidad de Chile. http://repositorio.uchile.cl/bitstream/handle/2250/108765/ gonzalez_r.pdf?sequence $=3 \&$ isAllowedy

Grijalva E., (2015). Investigación de violencia psicológica en contra de la mujer en el área de Huehuetenango [Tesis de licenciatura, Universidad Rafael Landívar]. Repositorio institucional de la Universidad Rafael Landívar. http://recursosbiblio.url.edu.gt/tesisjcem/2015/07/03/Grijalva-Edy.pdf

Guba, EG \& Lincoln YS. (1981). Effective evaluation: improving the usefulness of evaluation result drought responsive and naturalist approach. Jossey-Bass Publishers.

Guba, EG \& Lincoln, YS. (1982). Epistemological and methodological bases of naturalistic inquiry. Educ Tech Res Dev, 30(4), 233-52.

Hernández, R., Fernández, C., y Baptista, P. (2014). Metodología de la investigación. México, D.F.: McGraw-Hill / Interamericana.

Huerta, R., Bulnes M., Ponce C., Sotil A., Campos E. (2014). Depresión y ansiedad en mujeres en situación de maltrato en la relación de pareja según tipo de convivencia, en zonas urbano marginales de Lima. Theorema - UNMSM Volumen 1 - $\mathrm{N}^{\mathrm{o}}$ 1 - Junio. https://revistasinvestigacion.unmsm.edu.pe/index.php/Theo/article/ view/11945/10690

Husserl, E. (2008). La crisis de las ciencias europeas y la fenomenología trascendental. Buenos Aires: Prometeo Libros. https://profesorvargasguillen.files.wordpress. com/2013/08/crisis-de-las-ciencias-europeas-y-la-fenomenologc3ada-trascendentaltrad-julia-iribarne-krisis.pdf

Jaramillo J. (2011). Distorsiones cognitivas en víctimas de violencia intrafamiliar y sexual [Trabajo de Grado Académico, Universidad Central del Ecuador]. Repositorio digital de la Universidad Central del Ecuador. http:/www.dspace.uce.edu.ec/ bitstream/25000/1261/1/T-UCE-0007-8.pdf

Lakatos, I. (1978). Pruebas y refutaciones. La lógica del descubrimiento matemático. Alianza.

Lakatos, I. (1981). Matemáticas, ciencia y epistemología. Escritos Filosóficos, 2. Alianza.

Längle, A. (2013). La Fenomenología en la Práctica Psicoterapéutica: Una Introducción al Análisis Existencial Personal (AEP). http://episjournal.com/journal-2013/phenomenology-in-psychotherapeutic-praxis

Lincoln, YS.; Guba EG. (1985). Naturalistic Inquiry. Age Publications.

Lincoln, YS \& Guba, EG. (1986). But is it rigorous? Trustworthiness and authenticity in naturalistic evaluation. New Dir Program Eval, 30, 73-84. https://doi.org/10.1002/ $\underline{\text { ev. } 1427}$ 
López, S. (2013). Sobre las incidencias del método en el carácter crítico de las ciencias Sociales. Un análisis de la posición de Karl Popper. Revista Colombiana de Filosofía de la Ciencia, 13(26), 31-45. https://www.redalyc.org/articulo.oa?id=414/41429979002

Martínez-Romero, J. (2018). Consideraciones sobre la violencia doméstica desde la perspectiva de una Psicología fenomenológico-existencial. Rev. Nufen: Phenom. Interd. Belém, 10(3), 179-198. http://pepsic.bvsalud.org/scielo.php?script=sci arttext\&pid=S2175-25912018000300013\&lng=pt\&nrm=iso

Nóblega, M. y Muñoz, P. (2009). Una aproximación cualitativa a la violencia hacia la mujer en un asentamiento humano de Villa El Salvador. Instituto de Investigación de Psicología, Universidad de San Martín de Porres. Revista Liberabit: 15(2): 95-108.

Orbegoso, E. (2015). Manual de metodología de la investigación cualitativa. Lima.

Ortego, M., López S., Álvarez, M., y Aparicio, M. (2011). Ciencias Psicosociales I. La Psicología y el método científico. Universidad de Cantabria. https:/ocw.unican.es/ pluginfile.php/1420/course/section/1836/tema_01.pdf

Pavón, S. (2020). La Epojé Fenomenológica. Las nueve musas. https://www.lasnuevemusas.com/la-epoje-fenomenologica/

Paz-Gonzales, C., Pino-Ramírez, G. (2011). Tendencia en la investigación sobre violencia familiar. Interacción y Perspectiva Revista de Trabajo Social, 1(1), 20-31. dialnet. unirioja.es>descarga articulo

Piscoya, L. (2009a). El proceso de la investigación científica. Universidad Inca Garcilaso de la Vega.

Piscoya, L. (2009b). Tópicos de epistemología. Universidad Inca Garcilaso de la Vega.

Popper, K. (1980). La lógica de la investigación científica. Tecnos.

Ramírez, E. (2014). Introducción a la psicología. Tema 1. La naturaleza del conocimiento cientifico. http://www4.ujaen.es/ eramirez/Descargas/temal

Reichenbach, H. (1938). Experience and Prediction. Chicago University Press.

Sánchez, F. (2019). Fundamentos epistémicos de la investigación cualitativa y cuantitativa: consensos y disensos. Revista Digital de Investigación en Docencia Universitaria, 13(1), 102-122. https://dx.doi.org/10.19083/ridu.2019.644

Santandreu, R. (2012). El arte de no amargarse la vida. Oniro.

Strasser, S. (1967). Miseria y grandeza del “hecho”. Una meditación fenomenológica. http://23118.psi.uba.ar/academica/carrerasdegrado/psicologia/informacion_adicional/obligatorias/034_historia_2/Archivos/Strasser.pdf

Torres, A. (2014). Violencia de pareja: detección, personalidad y bloqueo de la hui$d a$ [Tesis doctoral, Universidad de Barcelona]. Repositorio digital Tesis Doctorals en Xarxa. https://www.tdx.cat/bitstream/handle/10803/313041/ATG_TESIS. pdf? sequence $=1 \&$ isAllowed $=y$

Vargas, G. (2011). La fenomenología y el ideal de la ciencia. Revista Coherencia, 8(15), 69-87. http://www.scielo.org.co/pdf/cohe/v8n15/v8n15a03.pdf 
Vásquez K., Herrera N., Medina M. (2016). Análisis cualitativo del significado de la violencia conyugal en mujeres del Municipio de Cajicá, Colombia. Tordesillas: Revista de investigación Multidisciplinar,11, pp. 5-14. https://dialnet.unirioja.es/servlet/ articulo?codigo $=5967148$

Villena, D. (2014). El círculo de Viena. Una nota histórica. Analítica, 8(8), 123-130. http:// cesfia.org.pe/villena/circulo_de_viena.pdf 